\title{
Coordinated Beamforming with Limited BS Cooperation for Multicell Multiuser MIMO Broadcast Channel
}

\author{
Kyong-Hoe Kim ${ }^{1}$, Jong-Ho Lee ${ }^{2}$, Chang-Hoon Lee ${ }^{1}$, Nam-Ryul Jeon ${ }^{1}$, and Seong-Cheol Kim ${ }^{1}$ \\ ${ }^{1}$ Institute of New Media and Communications, \\ School of Electrical Engineering and Computer Science, \\ Seoul National University, Seoul, Korea \\ \{khkim, lchjsa, jeon3115, sckim\}@maxwell.snu.ac.kr \\ ${ }^{2}$ School of Electrical and Computer Engineering, \\ Georgia Institute of Technology, Atlanta, Georgia, USA \\ jh0512.1ee@gmail.com
}

\begin{abstract}
The capacity of downlink multi-input multi-output (MIMO) systems is significantly limited by inter-cell co-channel interference (CCI). Improving the performance of cell edge users who suffers strong inter-cell CCI is still a crucial issue. In this paper, coordinated beamforming (CBF) algorithm for multicell multiuser MIMO system is proposed to cope with the presence of intel-cell CCI. In multicell environment with MIMO channel, limited base station (BS) cooperation is considered to design optimal transmit and receive beamformers which make interference-free at the receiver. Simulation results show that the proposed algorithms can improve the capacity of cell edge user effectively as well as the overall system capacity for multiuser multicell MIMO system.
\end{abstract}

Keywords-multiuser MIMO systetms; co-channel interference; interference suppression; BS cooperation

\section{INTRODUCTION}

Recently remarkable numbers of studies have done on the capacity of multiple-input multiple-output (MIMO) $[1,2]$. The optimal sum capacity of the MIMO broadcast channel given by information theory is achieved by dirty paper coding (DPC) [3]. Although DPC is theoretically optimal, it is difficult to implement in practice due to the significant amount of processing complexity [4]. For that reason, several practical techniques which approach the performance of DPC have been proposed. One of these approaches is block diagonalization (BD) [5] based on the precoding concept, which can transmit multiple data streams intended for each user simultaneously like DPC. Another promising algorithm is coordinated beamforming (CBF) which can nullify the inter-user interference allowing a larger number of receive antennas than the number of data streams per each receiver [6, 7]. In these techniques, the beamformer of each user is designed to be orthogonal to the channels of all other users so that the interuser interference is completely canceled. But, these techniques are based on a per-cell concept and they only consider interuser interference from different users in the same cell. Thus, inter-cell co-channel interference is treated as background noise in these techniques. However, in a cellular environment, co-channel interference $(\mathrm{CCI})$ that comes from adjacent cells becomes dominating channel impairment and degrades the overall system performance significantly.

This work is supported in part by Brain Korea 21 project and also supported in part by the Korea Research Foundation Grant funded by the Korean Government [KRF-2008-357-D00177].
To mitigate inter-cell CCI, a joint signal processing across different BSs is proposed in this paper. If the beamforming vectors for different transmitters and receivers in multiple cells are optimized jointly, the capacity of the multicell network is significantly improved. The motivation of an algorithm for inter-cell CCI cancellation comes from a recent study by Chae et al. [8]. They proposed an iterative optimization procedure to find the transmit beamforming and receive combining vectors which cancel inter-user interference in single-cell multiuser MIMO broadcast channel. In this paper, we modify and extend this algorithm with a particular type of BS cooperation to overcome not only the inter-user interference but also inter-cell $\mathrm{CCI}$ in multicell multiuser MIMO broadcast channel.

This paper is organized as follows. We introduce a multicell multiuser MIMO broadcast system model and the proposed multicell CBF algorithm with limited BS cooperation in Section II. In Section III, we present numerical results to show the sum rate performance of the proposed algorithm. Finally, conclusions and some insights are given in Section IV.

\section{SYSTEM MODEL AND PROBLEM FORMULATION}

\section{A. Multicell MIMO Broadcast System Model with BS Cooperation}

Suppose in general that there are a number of mobile stations (MSs) arbitrarily distributed in the downlink multicell MIMO system. At each cell, we design a wireless link equipped with $N_{t}$ transmit antenna elements at the BS and $N_{r}$ receive antenna elements at the users. There are $N_{b}$ independent co-channel adjacent BSs, in each of which $K$ MSs are independently selected based on the scheduler criterion for getting the data from the BS where we assume that the number of scheduled users is the same as the number of transmit antennas $\left(K=N_{T}\right)$, as described in Fig. 1 for a case with $N_{b}=2$. Among $K$ MSs in each independent cell, there are $L$ MSs which are selected by a single cell and $M$ MSs which are selected by multiple cells $(K=L+M)$. In other words, $M$ MSs are cell edge users (users having low Signal-to-Interference Ratio (SIR) due to weak signal and strong CCI) who suffer from severe inter-cell co-channel interference and $L$ MSs are center users (users that experience very low CCI and have high SIR). 


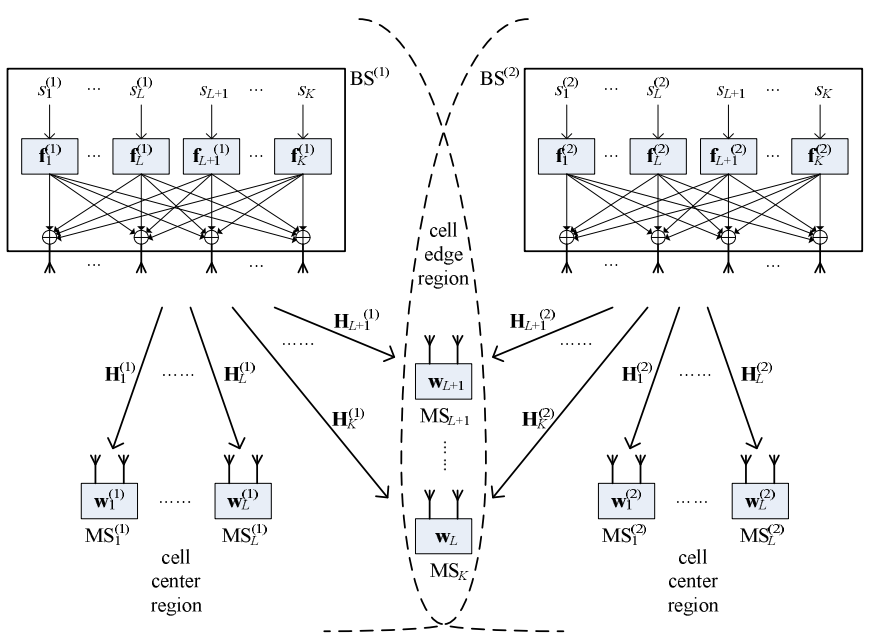

Figure 1. Multiuser Multicell MIMO Broadcast System Model with Limited BS Cooperation

In this paper, $M$ MSs are denoted to co-channel users and $L$ MSs are denoted to single-channel users.

We assume that the channel between the BS $i$ and user $k$ is represented by a matrix $\mathbf{H}_{k}^{(i)}$ of size $N_{r} \times N_{t}$ whose elements are i.i.d. complex Gaussian random variables with zero mean and unit variance and the channel matrix of a scheduled user $k$ is available at the BS as perfect channel state information (CSI) Note that in the rest of the paper the bracketed number in the superscript and the number in subscript indicate the index of the cell region (or BS itself) and the index of the user (MS) respectively. In Fig. 1, for example, $\mathrm{MS}_{L}^{(i)}$ indicates the $L$-th user located in cell region 1 and $\mathrm{MS}_{K}$ indicates the $K$-th user located in multicell (cell edge) region. For $M$ cell edge users' parameter, we put the subscript in order from $L+1$ to $K$ and the superscript is safely omitted because there are located in multicell region.

We assume that $L$ MSs in cell center do not suffer from inter-cell CCI. Therefore, there is no need to define channel matrix between MSs in cell center and BSs in adjacent cells.

Since cell edge users has worse channel SINR and far lower throughput compared to cell center users, we introduce BS cooperation into the downlink multicell MIMO system for throughput enhancement. For the traditional non-cooperative scenario with single cell signaling, a MS only communicates with its own BS. But, for the cooperative BS scenario with multicell signaling, adjacent BSs can jointly transmit data to MSs to increase throughput. $M$ MSs located in cell edge region receive jointly transmitted data in the proposed system for BS cooperation.

\section{B. Coordinated Beamforming for Multicell MIMO Broadcast} System

Let $s_{k}^{(i)}$ and $s_{k}$ denote the $k$-th transmit symbol designed to be transmitted to $\mathrm{MS}_{k}^{(i)}$ located in cell region $i$ and $\mathrm{MS}_{k}$ located in multicell region, respectively. In this system, the symbol $s_{k}$ is simultaneously transmitted from $N_{b}$ BSs for joint transmission. Using a similar index notation, $\mathbf{n}_{k}^{(i)}$ and $\mathbf{n}_{k}$ denote the $N_{r} \times 1$ additive white Gaussian noise vector with variance $\sigma_{k}^{(i) 2}$ and $\sigma_{k}^{2}$ per entry observed at the $\mathrm{MS}_{k}^{(i)}$ located in cell region $i$ and $\mathrm{MS}_{k}$ located in multicell region, respectively. Let $\mathbf{f}_{k}^{(i)}$ denote the $N_{t}$ $\times 1$ transmit beamformer vector (assumed unit norm, i.e. there is no power allocation) in the $\mathrm{BS}^{(i)}$ and $\mathbf{w}_{k}^{(i)}$ and $\mathbf{w}_{k}$ denote the $N_{r} \times 1$ combining vector (receive beamformer) for the $k$-th user. The same data symbol $s_{k}(L+1 \leq k \leq K)$ is transmitted from multiple adjacent BSs to a cell edge user for BS cooperation.

The received signal at the MS after combining is

$$
\begin{aligned}
y_{k}^{(i)}= & \mathbf{w}_{k}^{(i) H} \mathbf{H}_{k}^{(i)} \mathbf{f}_{k}^{(i)} s_{k}^{(i)}+\mathbf{w}_{k}^{(i) H} \mathbf{H}_{k}^{(i)}\left(\sum_{n=1, n \neq k}^{L} \mathbf{f}_{n}^{(i)} s_{n}^{(i)}+\sum_{n=L+1}^{K} \mathbf{f}_{n}^{(i)} s_{n}\right) \\
& +\mathbf{w}_{k}^{(i) H} \mathbf{n}_{k}^{(i)}, \text { for } 1 \leq k \leq L \\
y_{k}= & \mathbf{w}_{k}^{H} \sum_{i=1}^{N_{b}} \mathbf{H}_{k}^{(i)} \mathbf{f}_{k}^{(i)} s_{k}+\mathbf{w}_{k}^{H} \sum_{i=1}^{N_{b}} \mathbf{H}_{k}^{(i)}\left(\sum_{n=1}^{L} \mathbf{f}_{n}^{(i)} s_{n}^{(i)}+\sum_{n=L+1, n \neq k}^{K} \mathbf{f}_{n}^{(i)} s_{n}\right) \\
& +\mathbf{w}_{k}^{H} \mathbf{n}_{k}, \text { for } L+1 \leq k \leq K .
\end{aligned}
$$

Equation (1) and (2) is the received signal at the MS for $L$ cell center users of each cell and $M(=K-L)$ cell edge users, respectively. The first term, the last term, and the middle term in (1) and (2) is the desired signal, the noise, and the interference, respectively. To remove the interference term in (1) and (2), we introduce the CBF transmission strategies [8]. The BS chooses the transmit beamformer $\mathbf{f}_{k}^{(i)}$ such that

$$
\begin{aligned}
& \text { if } 1 \leq k \leq L, \quad \mathbf{w}_{j}^{(i) H} \mathbf{H}_{j}^{(i)} \mathbf{f}_{k}^{(i)}=0, \text { for } j=1, \cdots, k-1, k+1, \cdots, K \\
& \text { if } L+1 \leq k \leq K, \quad \mathbf{w}_{j}^{H} \mathbf{H}_{j}^{(i)} \mathbf{f}_{k}^{(i)}=0, \quad \text { for } j=1, \cdots, k-1, k+1, \cdots, K
\end{aligned}
$$

If $\mathbf{f}_{k}^{(i)}$ is chosen in this way, all interference terms are canceled out.

An equivalent channel matrix defined in $[6,7]$ can be easily extended to the multicell system as described below,

$$
\tilde{\mathbf{H}}_{k}^{(i)}=\left[\begin{array}{llllll}
\overline{\mathbf{h}}_{1}^{(i) T} & \ldots & \overline{\mathbf{h}}_{k-1}^{(i) T} & \overline{\mathbf{h}}_{k+1}^{(i) T} & \ldots & \overline{\mathbf{h}}_{K}^{(i) T}
\end{array}\right]^{T},
$$

where $\overline{\mathbf{h}}_{k}^{(i)}=\mathbf{w}_{k}^{(i) H} \mathbf{H}_{k}^{(i)}, \quad$ for $1 \leq k \leq L$ and $\overline{\mathbf{h}}_{k}^{(i)}=\mathbf{w}_{k}{ }^{H} \mathbf{H}_{k}^{(i)}$, for $L+1 \leq k \leq K$ and then transmit beamformers $\mathbf{f}_{k}^{(i)}$ are solved to satisfy $\tilde{\mathbf{H}}_{k}^{(i)} \mathbf{f}_{k}^{(i)}=0$. Therefore, (3) and (4) can be fulfilled, that is, each users received signal is interference free after combining.

If we assume that $K=N_{t}$ and the channels are sufficiently rich, $\tilde{\mathbf{H}}_{k}^{(i)}$ will be full-rank and of dimension $(K-1) \times K$ thus the null-space has dimension one and there is only one zero singular value.

Let the singular value decomposition (SVD) of $\tilde{\mathbf{H}}_{k}^{(i)}$ be

$$
\tilde{\mathbf{H}}_{k}^{(i)}=\tilde{\mathbf{U}}_{k}^{(i)} \tilde{\mathbf{D}}_{k}^{(i)}\left(\tilde{\mathbf{V}}_{k}^{(i)}\right)^{H}
$$

where $\tilde{\mathbf{U}}_{k}^{(i)}, \tilde{\mathbf{D}}_{k}^{(i)}$, and $\tilde{\mathbf{V}}_{k}^{(i)}$ is the left singular matrix, the matrix of singular values of $\tilde{\mathbf{H}}_{k}^{(i)}$, and the right singular matrix, 
respectively. As the null-space of $\tilde{\mathbf{H}}_{k}^{(i)}$ has dimension one, the last $K$-th column vector $\tilde{\mathbf{v}}_{K, k}^{(i)}$ of the right singular matrix $\tilde{\mathbf{V}}_{k}^{(i)}$ has to be a vector corresponding to zero singular value, that is, $\tilde{\mathbf{H}}_{k}^{(i)} \tilde{\mathbf{v}}_{K, k}^{(i)}=\mathbf{0}$. So, the transmit beamformer $\mathbf{f}_{k}^{(i)}$ is

$$
\mathbf{f}_{k}^{(i)}=\tilde{\mathbf{v}}_{K, k}^{(i)}
$$

In this paper, we assume that maximum ratio combining (MRC) is used at the receiver and then the combining vector is given by

$$
\begin{array}{ll}
\mathbf{w}_{k}^{(i)}=\mathbf{H}_{k}^{(i)} \mathbf{f}_{k}^{(i)} & \text { for } 1 \leq k \leq L, \\
\mathbf{w}_{k}=\sum_{i=1}^{N_{b}} \mathbf{H}_{k}^{(i)} \mathbf{f}_{k}^{(i)} & \text { for } L+1 \leq k \leq K .
\end{array}
$$

To calculate MRC at each receiver, $\tilde{\mathbf{H}}_{k}^{(i)}$, that is, CSIs of other users in the cell region $i$ (including the cell edge region), must be needed at each receiver. Instead, by sending the beamforming information $\mathbf{f}_{k}^{(i)}$ from $\mathrm{BS}^{(i)}$ to each receiver, the receiver can estimate combining vector using MRC. So, computations of optimal transmit and receive beamforming vectors are totally performed at the BS. Quantized transmit beamforming, introduced as limited feedforward information, was carefully studied in [8]. In this paper, the quantization is not considered for simplicity.

Using the solution stated above, the proposed iterative algorithm is formulated as follows. Note that the notation $(j)$ denotes the $j$-th iteration.

\section{Step.1 Initialization:}

- $\quad$ For $\forall i, 1 \leq k \leq K$, compute $\mathbf{H}_{k}^{(i)}=U_{k}^{(i)} D_{k}^{(i)}\left(V_{k}^{(i)}\right)^{H}$, then the initial receive beamforming vector is set to the principle singular vector of the left singular matrix.

$$
\begin{array}{ll}
\mathbf{w}_{k}^{(i)}(1)=\mathbf{u}_{1, k}^{(i)} & (1 \leq k \leq L) \\
\mathbf{w}_{k}(1)=\mathbf{u}_{1, k}^{(i)} & (L+1 \leq k \leq K, i \text { is randomly selected })
\end{array}
$$

\section{Step.2 Iterative Update:}

- For $\forall i, 1 \leq k \leq K$, compute the effective channel vectors as follows

$$
\begin{aligned}
& \overline{\mathbf{h}}_{k}^{(i)}=\mathbf{w}_{k}^{(i) H}(j-1) \mathbf{H}_{k}^{(i)} \quad(1 \leq k \leq L) \\
& \overline{\mathbf{h}}_{k}^{(i)}=\mathbf{w}_{k}{ }^{H}(j-1) \mathbf{H}_{k}^{(i)} \quad(L+1 \leq k \leq K)
\end{aligned}
$$

- Compute the equivalent channel matrix as follows

$$
\begin{aligned}
\tilde{\mathbf{H}}_{k}^{(i)} & =\left[\begin{array}{llllll}
\overline{\mathbf{h}}_{1}^{(i) T} & \cdots & \overline{\mathbf{h}}_{k-1}^{(i) T} & \overline{\mathbf{h}}_{k+1}^{(i) T} & \cdots & \overline{\mathbf{h}}_{K}^{(i) T}
\end{array}\right]^{T} \\
\tilde{\mathbf{H}}_{k}^{(i)} & =\tilde{\mathbf{U}}_{k}^{(i)} \tilde{\mathbf{D}}_{k}^{(i)}\left(\tilde{\mathbf{V}}_{k}^{(i)}\right)^{H}
\end{aligned}
$$

- Solve and Update for transmit and receive beamformers

$$
\begin{aligned}
& \mathbf{f}_{k}^{(i)}(j)=\tilde{\mathbf{v}}_{K, k}^{(i)}, \\
& (1 \leq k \leq K) \\
& \mathbf{w}_{k}^{(i)}(j)=\mathbf{H}_{k}^{(i)} \mathbf{f}_{k}^{(i)}(j), \quad(1 \leq k \leq L) \\
& \mathbf{w}_{k}(j)=\sum_{i=1}^{N_{b}} \mathbf{H}_{k}^{(i)} \mathbf{f}_{k}^{(i)}(j), \quad(L+1 \leq k \leq K)
\end{aligned}
$$

- If $\left\|\mathbf{f}_{k}^{(i)}(j)-\mathbf{f}_{k}^{(i)}(j-1)\right\|<\varepsilon, \forall i$, break. Otherwise, increase the index as $j=j+1$ and repeat Step. 2

Final TX/RX Beamformers:

$$
\begin{array}{ll}
\mathbf{f}_{k}^{(i)}=\mathbf{f}_{k}^{(i)}(j), & (1 \leq k \leq K) \\
\mathbf{w}_{k}^{(i)}=\mathbf{w}_{k}^{(i)}(j), & (1 \leq k \leq L) \\
\mathbf{w}_{k}=\mathbf{w}_{k}(j), & (L+1 \leq k \leq K)
\end{array}
$$

\section{Sum Rate Analysis}

When the transmit beamforming and receive combining vectors are computed by the proposed algorithms, there is no inter-cell CCI as well as no remaining interference between the receivers. Therefore, the effective MIMO channel matrix, observed by the transmitter has only diagonal elements, $\mathbf{w}_{k}^{(i) H} \mathbf{H}_{k}^{(i)} \mathbf{f}_{k}^{(i)}$ or $\mathbf{w}_{k}^{H} \sum_{i=1}^{N_{b}} \mathbf{H}_{k}^{(i)} \mathbf{f}_{k}^{(i)}$ for $1 \leq k \leq L$ or $L+1 \leq k \leq K$, respectively.

The achievable sum rate of cell $i$ including cell edge region is given by

$$
\begin{aligned}
R_{\text {sum }}^{(i)} & =\sum_{k=1}^{L} \log _{2}\left(1+\frac{P_{k}^{(i)}}{\sigma_{k}^{(i) 2}}\left|\mathbf{w}_{k}^{(i) H} \mathbf{H}_{k}^{(i)} \mathbf{f}_{k}^{(i)}\right|^{2}\right) \\
& +\sum_{k=L+1}^{K} \log _{2}\left(1+\sum_{i=1}^{N_{b}} \frac{P_{k}^{(i)}}{\sigma_{k}^{2}}\left|\mathbf{w}_{k}^{H} \mathbf{H}_{k}^{(i)} \mathbf{f}_{k}^{(i)}\right|^{2}\right)[\mathrm{bps} / \mathrm{Hz}],
\end{aligned}
$$

where $\sigma_{k}^{(i) 2}$ and $\sigma_{k}^{2}$ are the AWGN variance at the $\mathrm{MS}_{k}^{(i)}$ located in cell region $i$ and $\mathrm{MS}_{k}$ located in multicell region, respectively, and $P_{k}^{(i)}$ is the transmit power allocated to the $k$-th transmit symbol of the $\mathrm{BS}^{(i)}$. by

The achievable sum rate of co-channel cells is then given

$$
\begin{aligned}
R_{\text {sum }} & =\sum_{i=1}^{N_{b}} \sum_{k=1}^{L} \log _{2}\left(1+\frac{P_{k}^{(i)}}{\sigma_{k}^{(i) 2}}\left|\mathbf{w}_{k}^{(i) H} \mathbf{H}_{k}^{(i)} \mathbf{f}_{k}^{(i)}\right|^{2}\right) \\
& +\sum_{k=L+1}^{K} \log _{2}\left(1+\sum_{i=1}^{N_{b}} \frac{P_{k}^{(i)}}{\sigma_{k}^{2}}\left|\mathbf{w}_{k}^{H} \mathbf{H}_{k}^{(i)} \mathbf{f}_{k}^{(i)}\right|^{2}\right)[\mathrm{bps} / \mathrm{Hz}] .
\end{aligned}
$$

The maximum achievable sum rate at each cell is obtained through classical water-filling, but we assume equal power allocation for simplicity in this paper.

\section{NUMERICAL RESULTS}

In this section, we show the performance of the proposed beamforming scheme in terms of an achievable sum rate of co- 


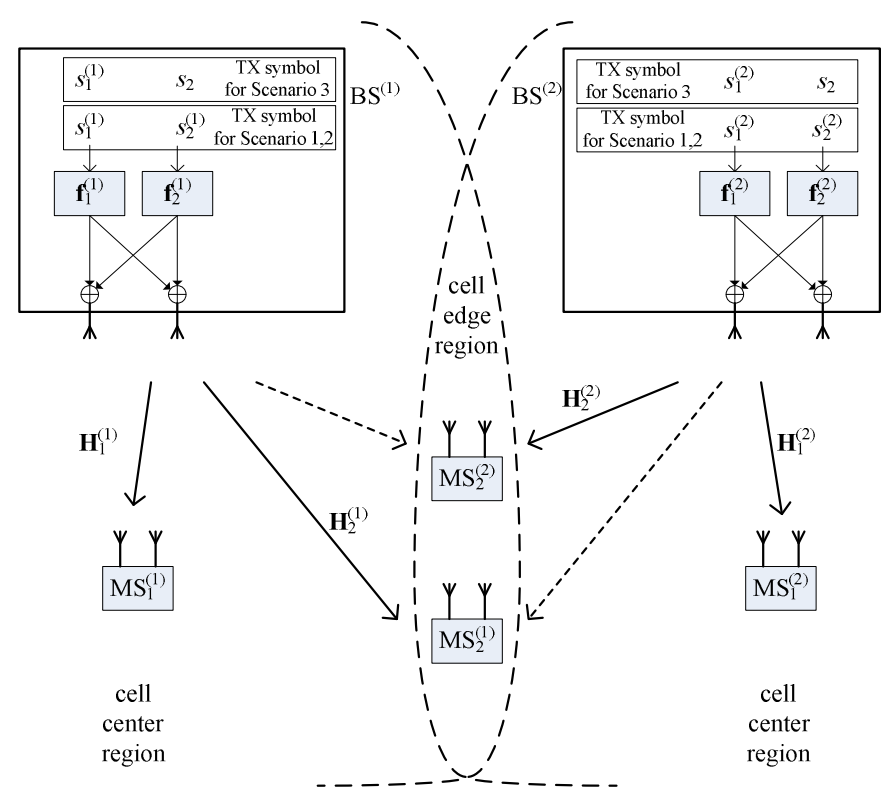

Figure 2. Simple Multiuser Multicell MIMO System Model for Performance Comparison

channel cells. To show the improved performances of the sum rate with the proposed algorithm, we set up a simple multiuser multicell MIMO broadcast network. We compare the sum rate of the proposed multicell CBF algorithm with limited BS cooperation and the CBF algorithm [8] with or without BS cooperation. Then, we show that the capacity of cell edge user is improved when the number of adjacent cells is increased.

\section{A. Performance Comparison on a Simple Multiuser Multicell MIMO Broadcast Network}

A simple two-cell configuration, $\left(M, K, N_{b}\right)=(1,2,2)$ multiuser MIMO network with one co-channel user located between two adjacent cells (Fig. 2) is considered. We also assume that $K=N_{t}=N_{r}=2$ as described in Fig. 2. Although we assume only one co-channel user $(M=1)$, there are actually two co-channel user, $\mathrm{MS}_{2}^{(1)}$ and $\mathrm{MS}_{2}^{(2)}$ in Fig. 2. In the simulation with the proposed multicell CBF algorithm with limited BS cooperation, the proposed algorithm can support only one co-channel user (and each single-channel user of two cells, $\mathrm{MS}_{1}^{(1)}$ and $\mathrm{MS}_{1}^{(2)}$ ), so we decide to choose just one cochannel user that has higher achievable rate. Instead, in the simulation with the CBF algorithm with no BS cooperation or full $\mathrm{BS}$ cooperation, we assume that $\mathrm{MS}_{2}^{(1)}$ is belong to $\mathrm{BS}^{(1)}$ and $\mathrm{MS}_{2}^{(2)}$ is belong to $\mathrm{BS}^{(2)}$, because this algorithm can't have multicell concept. Three different scenarios for the simulation are described in detail as follows.

\section{Scenario 1) CBF algorithm with no BS cooperation}

$\mathrm{BS}^{(1)}$ transmits symbol $s_{1}^{(1)}$ and $s_{2}^{(1)}$ for $\mathrm{MS}_{1}^{(1)}$ and $\mathrm{MS}_{2}^{(1)}$, respectively. In the same manner, $\mathrm{BS}^{(2)}$ transmits symbol $s_{1}^{(2)}$ and $s_{2}^{(2)}$ for $\mathrm{MS}_{1}^{(2)}$ and $\mathrm{MS}_{2}^{(2)}$, respectively. Dashed arrows in Fig. 2 represent the presence of inter-cell CCI.

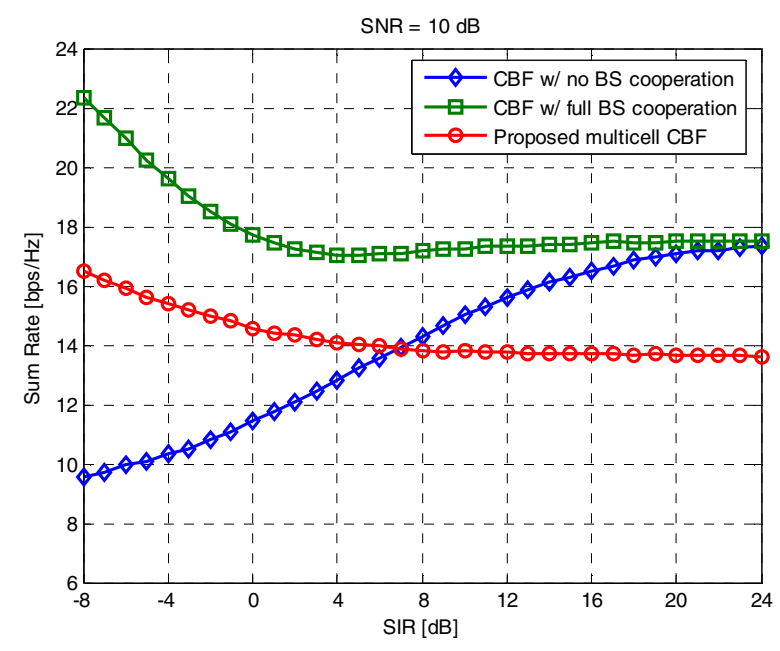

Figure 3. Sum Rate Comparison $\left(M, K, N_{b}\right)=(1,2,2)$

\section{Scenario 2) CBF algorithm with full BS cooperation}

$\mathrm{BS}^{(1)}$ and $\mathrm{BS}^{(2)}$ transmits exactly the same symbol with scenario 1. Dashed arrows also represent the presence of intercell CCI. Only the difference with scenario 1 is an introduction of full BS cooperation. Two BSs must share the CSI and the transmit data of all MSs located in their cell region for full BS cooperation. Then, the BSs can compute TX and RX beamforming vectors with single-cell $\mathrm{CBF}$ algorithm using shared CSI and data. Therefore, the BSs must be connected to a high-speed wired backbone that allows information to be reliably exchanged between them. Conceptually, they act as a single BS which has $2 N_{t}$ transmit antennas. So, all the interference can be canceled by CBF algorithm.

Scenario 3) Proposed multicell CBF algorithm with limited BS cooperation

$\mathrm{BS}^{(1)}$ transmits symbol $s_{1}^{(1)}$ and $s_{2}$, and $\mathrm{BS}^{(2)}$ transmits symbol $s_{1}^{(2)}$ and $s_{2}$. Here, $s_{2}$ is jointly transmitted from both two BSs to a co-channel user $\left(\mathrm{MS}_{1}^{(2)}\right.$ or $\left.\mathrm{MS}_{2}^{(2)}\right)$. All the interference can be canceled by the proposed multicell CBF algorithm. Two BSs can compute TX and RX beamforming vectors using the CSI of all MSs located in their cell region and jointly transmitted symbol $s_{2}$. Although all BSs must know the CSI of all MSs like full BS cooperation, two BSs share only a symbol $\left(s_{2}\right)$, which is transmitted from two BSs jointly, instead of all transmit symbols of $\mathrm{BS}^{(1)}$ and $\mathrm{BS}^{(2)}$ in case of full $\mathrm{BS}$ cooperation. This is why we call it a limited BS cooperation compared with a full BS cooperation.

Fig. 3 shows sum rates for 1) $\mathrm{CBF}$ algorithm with no $\mathrm{BS}$ cooperation, 2) $\mathrm{CBF}$ algorithm with full BS cooperation, and 3) Proposed multicell CBF algorithm with limited BS cooperation, where the number of receiver antennas $N_{r}$ is fixed to 2 , the Signal-to-Noise Ratio (SNR) is fixed to $10 \mathrm{~dB}$, and the Signalto-Interference Ratio (SIR) is varied within practically interested ranges. 


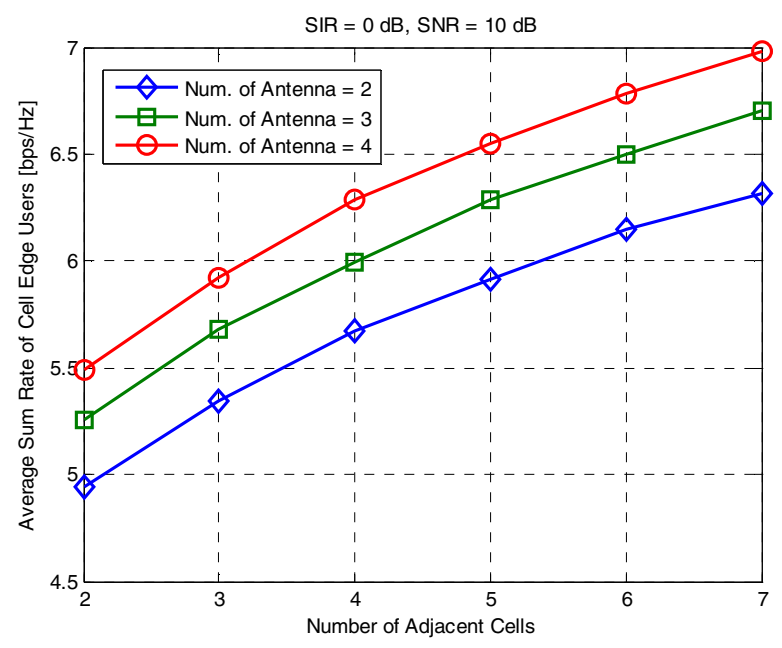

Figure 4. Average sum rate of cell edge users

Reasonably, CBF algorithm with full BS cooperation shows the best sum rate capacity, because the full BS cooperation with the knowledge of downlink CSI makes the network interference-free at the expense of more complex structure. $\mathrm{CBF}$ algorithm with no BS cooperation suffers from severe inter-cell CCI with low SIR. As the SIR is increased, the inter-cell CCI is decreased in the network, and the sum rate of Scenario 2 approximates the sum rate of scenario 1 . There is a significant performance gap between the sum rates of Scenario 2 and 3 in environments with strong interference (SIR of $6 \mathrm{~dB}$ or less). When the SIR is very low, the proposed multicell CBF algorithm with limited BS cooperation (Scenario 3) cancels the inter-cell CCI out and outperforms Scenario 1, although a joint transmission of transmit symbols decreases the sum rate compared to Scenario 2.

\section{B. Capacity of Cell Edge Users}

In this section, we examine the capacity of cell edge users obtained through the techniques we have developed in the last section (Scenario 3). Fig. 4 shows the average sum rate of $M=$ 2 cell edge users with respect to the number of adjacent cells, $N_{b}$ and the number of transmit antennas $N_{t}$ when cell edge users suffer from severe inter-cell CCI (SIR is fixed to $0 \mathrm{~dB}$ in this simulation). In this simulation, the number of receiver antennas $N_{r}$ is fixed to 2. From the figure it can be seen that as the number of adjacent cells grows larger, the average sum rate of cell edge users increases, too. In other words, the number of participating base stations increases the capacity of cell edge users increases. This extra gain comes from multicell diversity (or macrodiversity) introduced by BS cooperative multicell processing. This macrodiversity comes from a situation where several transmitters are used for transferring the same signal. As the number of transmit antennas grows larger, the average sum rate of cell edge users also increases. This extra gain obviously comes from transmit diversity.

\section{CONCLUSION}

In the next generation mobile communication system, intercell interference has become the major obstacle to achieve high spectral efficiency. In this paper, a multicell coordinated beamforming algorithm with limited BS cooperation is proposed for multicell multiuser MIMO broadcast channel. This algorithm can cope with not only the inter-user interference but also inter-cell CCI. To mitigate inter-cell CCI, a joint signal processing across different BSs is proposed. Simulation results show that the proposed multicell CBF algorithm outperform the conventional single-cell $\mathrm{CBF}$ algorithm in environments with strong inter-cell interference and the proposed algorithms can improve the capacity of cell edge user effectively as well as the overall system capacity for multiuser multicell MIMO system. As a proposed limited BS cooperation scheme introduces multi-cell diversity, the average sum rate of cell edge users increases with respect to the number of adjacent cells.

\section{REFERENCES}

[1] E. Telatar, "Capacity of multi-antenna Gaussian channels," Eur. Trans. Telecommun., vol. 10, pp. 585-598, Nov. 1999.

[2] G. J. Foschini and M. J. Gans, "On limits of wireless communications in a fading environment when using multiple antennas," Wireless Pers. Commun., vol. 6, pp. 311-335, Mar. 1998.

[3] M. Costa, "Writing on dirty paper," IEEE Trans. Inf. Theory, vol. 29, pp. 439-441, May 1983.

[4] G. Caire and S. Shamai (Shitz), "On the achievable throughput of a multi-antenna Gaussian broadcast channel," IEEE Trans. Inf. Theory, vol. 43, pp. 1691-1706, Jul. 2003.

[5] Q. H. Spencer, A. L. Swindlehurst, and M. Haardt, "Zero-forcing methods for downlink spatial multiplexing in multiuser MIMO channels," IEEE Trans. Signal Processing, vol. 52, no. 2, pp. 461-471, Feb. 2004.

[6] B. Farhang-Boroujeny, Q. Spencer, and A. L. Swindlehurst, "Layering techniques for space-time communications in multi-user networks," Proc. IEEE Veh. Technol. Conf., vol. 2, pp. 1339-1342, Oct. 2003.

[7] Z. Pan, K.-K. Wong, and T.-S. Ng, "Generalized multiuser orthogonal space-division multiplexing," IEEE Trans. Wireless Comm., vol. 3, pp. 1969-1973, Nov. 2004.

[8] C.-B. Chae, D. Mazzarese, and R. W. Heath Jr., "Coordinated beamforming for multiuser MIMO systems with limited feedforward," Proc. of Asilomar Conf. on Sign., Syst. and Computers, pp. 1511-1515, Oct.-Nov. 2006. 